03

\title{
Аномальный отклик стратифицированной жидкости на механическое воздействие, обусловленный термодиффузией
}

\author{
(С) Л.Х. Ингель \\ Научно-производственное объединение „Тайфун“, \\ 249038 Обнинск, Россия \\ Институт физики атмосфреры им. А.М. Обухова РАН, \\ 119017 Москва, Россия \\ e-mail: lev.ingel@gmail.com
}

(Поступило в Редакцию 24 мая 2017 г.)

В линейном приближении аналитически исследованы стационарные возмущения, вносимые в стратифицированную по температуре жидкую бинарную смесь (например, соленую воду) неоднородными касательными напряжениями на ее поверхности. Показано, что эффект термодиффузии может приводить к резкому увеличению глубины проникновения термических возмущений в среду, несмотря на устойчивую фоновую стратификацию плотности.

DOI: 10.21883/JTF.2018.01.45474.2350

\section{Введение}

Если к поверхности жидкости приложены неоднородные механические касательные напряжения, то в приповерхностном слое возникают горизонтальнонеоднородные течения; из соображений неразрывности это означает возникновение вертикальных движений. В вертикально стратифицированной по температуре среде при этом возникают термические неоднородности. Подобные задачи имеют важные приложения, в частности в геофизике, например, задача расчета отклика верхнего слоя водоемов на неоднородные ветровые воздействия [1]. Если стратификация плотности достаточно устойчива, то глубина проникновения упомянутых неоднородностей в среду относительно мала по сравнению с характерными горизонтальными масштабами неоднородных напряжений $L$. В простейшем случае гармонических по горизонтали неоднородностей, когда $L=2 \pi / k$, где $k-$ волновое число, характерный вертикальный масштаб возмущений составляет [1] $h \sim\left(\nu \kappa / k^{2} \alpha g \gamma_{T}\right)^{1 / 6}=\left(\nu \kappa / N_{T}^{2} k^{2}\right)^{1 / 6}$, где $v-$ кинематический коэффициент вязкости, $\kappa-$ коэффициент температуропроводности, $g$ - ускорение свободного падения, $\alpha$ - термический коэффициент расширения среды, $\gamma_{T}-$ фоновый вертикальный градиент температуры, $N_{T}=\left(\alpha g \gamma_{T}\right)^{/ 2}$ - частота Брента-Вяйсяля (частота плавучести). В распространенных ситуациях значения аналога числа Рэлея $N_{T}^{2} / v \kappa k^{4}$ велики, так что $h \ll L$, т. е. речь идет о возмущениях, проникающих лишь в весьма тонкий верхний слой среды.

В работах [2,3] было обращено внимание на то, что в двухкомпонентных средах (плотность которых зависит как от температуры, так и от концентрации примеси, например, в соленой морской воде) ситуация может радикально меняться - глубина проникновения термических возмущений может возрастать на порядки и достигать порядка горизонтального масштаба неод- нородностей $L$. Но необходимое условие этого нетривиального эффекта - наличие фоновой стратификации концентрации примеси, т. е. достаточно заметного вклада примеси в фоновую стратификацию плотности.

В упомянутых работах использовано распространенное приближение, в котором не учитывается термодиффузия (эффект Соре, см., например, [4-6]) — влияние температурных неоднородностей на перенос примеси. В настоящей работе показано, что при учете даже относительно слабой термодиффузии, присущей, например, соленой морской воде, глубина проникновения термических возмущений в среду может резко возрастать и при отсутствии фоновой стратификации примеси.

\section{Постановка задачи}

Геометрия задачи схематически изображена на рис. 1 . Рассматривается полуограниченный слой среды $z \leq 0$ (ось $z$ направлена вертикально вверх), устойчиво стратифицированный по температуре. Фоновая стратификация концентрации примеси (для определенности будем говорить о соленой воде) в отличие от [2,3] отсутствует.

Линеаризованная стационарная система уравнений для возмущений в приближении Буссинеска с учетом

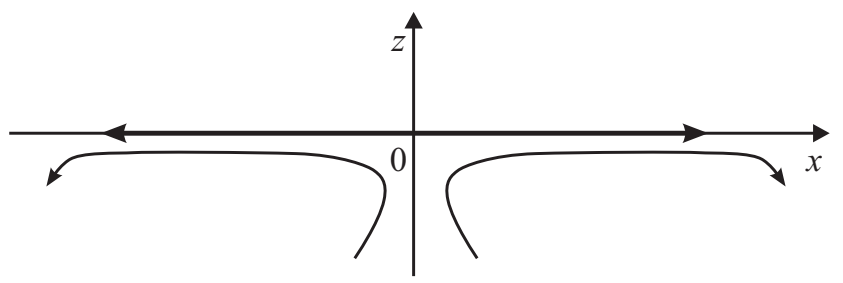

Рис. 1. Геометрия задачи. Стрелками схематически обозначены неоднородные касательные напряжения на горизонтальной поверхности жидкости и линии тока возникающих течений. 
термодиффузии, имеет вид [2-6]

$$
\begin{gathered}
0=-\frac{1}{\rho_{0}} \nabla p+v \nabla^{2} \mathbf{v}+g(\alpha T-\beta s) \mathbf{e}_{z}, \quad \nabla \mathbf{v}=0, \\
\gamma_{T} \mathbf{v e}_{z}=\kappa \nabla^{2} T, \quad 0=\chi \nabla^{2} s+\chi_{T} \nabla^{2} T .
\end{gathered}
$$

Здесь $T$ и $s$ - возмущения температуры и концентрации примеси (солености, промилле) соответственно, $\mathbf{v}-$ вектор возмущения поля скорости, $p-$ возмущение давления, $\mathbf{e}_{z}-$ единичный вектор в направлении вертикальной оси $z, \rho_{0}-$ отсчетная плотность среды, $\chi-$ коэффициент диффузии примеси, $\chi_{T}-$ соответствующий коэффициент, относящийся к термодиффузии, $\beta-$ коэффициент соленостного сжатия среды.

Поясним последнее из уравнений (1) (остальные достаточно традиционны). В более общем случае стационарное уравнение переноса примеси имеет вид [5]

$$
0=\nabla\left[\chi \nabla c+\chi s_{T} c\left(1-\frac{c}{\rho}\right) \nabla T\right] .
$$

Здесь $c$ - концентрация примеси $\left(\mathrm{kg} / \mathrm{m}^{3}\right), \rho-$ плотность среды, $T-$ температура, $s_{T}-$ коэффициент Cope $\left({ }^{\circ} \mathrm{C}\right)^{-1}[4,5]$. Ограничиваемся рассмотрением возмущений, проникающих в среду не слишком глубоко рассматриваем не слишком большие значения горизонтальных масштабов неоднородностей $L$ (в толстых слоях стационарное решение устанавливается очень долго ввиду медленности диффузии), когда можно пренебречь вариациями величин $\chi, s_{T}$ и $\rho$ (так что последнюю заменяем на $\rho_{0}$ ). В этом случае уравнение (2) можно переписать в виде

$$
\chi \nabla^{2} \mu+\chi s_{T} \nabla[\mu(1-\mu)] \nabla T+\chi s_{T} \mu(1-\mu) \nabla^{2} T=0,
$$

где введено обозначение $\mu=c / \rho_{0}-$ массовая доля примеси. Концентрация примеси и температура складываются из фоновых значений и возмущений. При линеаризации по возмущениям первое слагаемое имеет вид $\chi \nabla^{2} \mu^{\prime}$; порядок второго слагаемого $-\chi s_{T} \gamma_{T} \nabla \mu^{\prime}$ (здесь штрихом обозначены возмущения). Отношение абсолютной величины второго слагаемого к первому, как видно из масштабного анализа, порядка $l s_{T} \gamma_{T}$, где $l$ - наименьший из рассматриваемых пространственных масштабов возмущений. Ниже будут рассматриваться значения параметров, при которых упомянутое отношение много меньше единицы. Поэтому пренебрегаем вторым слагаемым и приходим к последнему уравнению (1), в котором

$$
\chi_{T}=10^{3} \chi s_{T} \bar{\mu}(1-\bar{\mu}),
$$

где чертой обозначено фоновое значение, а множитель $10^{3}$ связан с тем, что возмущение концентрации примеси $s$ здесь измеряется в промилле.
Предполагаем, что на поверхности жидкости $z=0$ заданы стационарные касательные напряжения, гармонически зависящие от горизонтальной координаты $x$ (ограничиваемся двумерной задачей на плоскости $(x, z))$ :

$$
\rho_{0} v \frac{\partial u}{\partial z}=E \sin k x \quad \text { при } \quad z=0,
$$

где $E-$ амплитуда напряжения, $u-$ горизонтальная составляющая скорости, $k-$ волновое число. Пренебрегаем деформациями поверхности, так что вертикальная составляющая скорости $w$ на поверхности $z=0$ обращается в нуль. Для отклонений температуры и концентрации примеси на поверхности предполагается выполнение краевых условий третьего рода:

$$
\frac{\partial T}{\partial z}=-\frac{T}{h_{T}}, \quad \frac{\partial s}{\partial z}=-\frac{s}{h_{s}}, \quad \text { при } \quad z=0,
$$

где неотрицательные величины $h_{T}, h_{s}$ - заданные масштабы длины. Предполагаем, что вдали от поверхности (при $z \rightarrow-\infty$ ) все возмущения затухают.

\section{Решение и его анализ}

Исключая $T$ из последних двух уравнений (1), получаем уравнение

$$
w \gamma_{s}=\chi \nabla^{2} s
$$

где введено обозначение

$$
\gamma_{s} \equiv-\frac{\gamma_{T} \chi_{T}}{\kappa}=-10^{3} \frac{\chi}{\kappa} \gamma_{T} s_{T} \bar{\mu}(1-\bar{\mu}) .
$$

Уравнение (7) формально совпадает с рассматриваемым в $[2,3]$ линеаризованным уравнением переноса примеси, где не учитывается термодиффузия, но предполагается наличие фоновой вертикальной стратификации примеси (фоновый вертикальный градиент концентрации примеси в этих работах обозначался через $\left.\gamma_{s}, \mathrm{ppm} / \mathrm{m}\right)$. Иными словами, задача, в которой последнее уравнение (1) можно теперь заменить на (7), свелась к ранее исследованной. Поэтому достаточно лишь вкратце напомнить решение.

Ищем для возмущений стационарные решения, гармонически зависящие от горизонтальной координаты $x$ :

$$
\begin{array}{ll}
u(x, z)=U(z) \sin (k x), & w(x, z)=W(z) \cos (k x), \\
T(x, z)=\theta(z) \cos (k x), & s(x, z)=\vartheta(z) \cos (k x)
\end{array}
$$

и т.д. Исключая из системы уравнений все неизвестные, кроме $w$, нетрудно получить уравнение

$$
\left(\frac{d^{2}}{d z^{2}}-k^{2}\right)^{3} W=k^{6} \mathrm{R} W
$$

где

$$
\mathrm{R}=\frac{1}{k^{4} \nu}\left(\frac{N_{T}^{2}}{\kappa}+\frac{N_{s}^{2}}{\chi}\right)=\mathrm{R}_{T}(1-\xi)=\mathrm{R}_{T}+\frac{\mathrm{R}_{s}}{\delta} .
$$


Здесь величины $N_{T}^{2}=\alpha g \gamma_{T}$ и $N_{s}^{2}=-\beta g \gamma_{s}$ имеют смысл квадратов частот плавучести, обусловленных стратификациями температуры и примеси соответственно; $\mathrm{R}_{T}=N_{T}^{2} / v \kappa k^{4}, \mathrm{R}_{s}=N_{s}^{2} / v \kappa k^{4}-$ аналоги чисел Рэлея,

$$
\xi \equiv \frac{\kappa \beta \gamma_{s}}{\chi \alpha \gamma_{T}}=-\frac{\kappa}{\chi} \frac{N_{s}^{2}}{N_{T}^{2}}=-\frac{1}{\delta} \frac{N_{s}^{2}}{N_{T}^{2}}, \quad \delta=\frac{\chi}{\kappa} .
$$

Безразмерный параметр $\mathrm{R}$ имеет смысл некоторого обобщенного числа Рэлея. Для рассматриваемой области допустимых значений $\gamma_{T}$ и $\gamma_{s}$ (предполагается гидростатическая устойчивость среды) он неотрицателен.

Ищем решение уравнения (9) в виде линейной комбинации экспонент $\exp \left(q_{i} k z\right)$, где $q_{i}-$ корни характеристического уравнения

$$
\left(q^{2}-1\right)^{3}=\mathrm{R}
$$

Из этих шести экспонент лишь три затухают при $z \rightarrow-\infty$; коэффициенты при остальных следует положить равными нулю. Поэтому решение для вертикальной скорости можно представить в виде

$$
W(z)=\sum_{i=1}^{3} C_{i} \exp \left(q_{i} k z\right),
$$

$$
q_{1}=\left(1+\mathrm{R}^{1 / 3}\right)^{1 / 2}, \quad q_{2,3}=\left[1+\mathrm{R}^{1 / 3} \exp \left( \pm \frac{2}{3} \pi i\right)\right]^{1 / 2} .
$$

Здесь $C_{i}$ - постоянные интегрирования, $\operatorname{Re} q_{i}>0$. Ниже ограничимся анализом решения для случая $\mathrm{R} \gg 1$ (точнее, предполагается выполнение более сильного неравенства $\mathrm{R}^{1 / 6} \gg 1$ ). Это соответствует ситуациям, когда устойчивая стратификация плотности достаточно существенна и в большой степени определяет структуру возникающих в среде возмущений. В таких случаях можно пренебречь единицей по сравнению с другими слагаемыми в выражениях (12), (14)

$$
q_{1} \approx \mathrm{R}^{1 / 6}, \quad q_{2,3} \approx \mathrm{R}^{1 / 6} \exp \left( \pm \frac{1}{3} \pi i\right)
$$

и решение заметно упрощается. В показателях экспонент в (13) (а также в выражениях для $u$ и возмущений давления и плавучести) присутствует большой множитель $\mathrm{R}^{1 / 6}$, так что возмущения упомянутых величин быстро затухают с глубиной по сравнению с их характерным горизонтальным масштабом $L=2 \pi / k$. Иными словами, аспектное отношение (отношение характерного горизонтального масштаба к вертикальному) у этих возмущений много больше единицы.

В выражениях для возмущений температуры и концентрации примеси по сравнению с (13), как легко видеть, вообще говоря, имеется и четвертая экспонента $\exp (k z)$. Постоянные интегрирования (коэффициенты при экспонентах) находятся из краевых условий. В аналитическое решение, приведенное в [2], вкрались отдельные опечатки; хотя они и не повлияли на основные результаты [2], имеет смысл привести здесь исправленное решение (за небольшими исключениями сохраняем те же обозначения, что и в [2]):

$$
\begin{aligned}
u= & -\mathrm{R}^{1 / 6} e^{K z}\left[C_{1} e^{K z}-2\left(C_{1}+\frac{\sqrt{3}}{4} \tau\right) \cos \sqrt{3} K z\right. \\
& \left.-\frac{\tau}{2} \sin \sqrt{3} K z\right] \sin (k x), \\
w= & e^{K z}\left[C_{1}\left(e^{K z}-\cos \sqrt{3} K z\right)-\left(\sqrt{3} C_{1}+\tau\right) \sin \sqrt{3} K z\right] \\
& \times \cos (k x), \\
T= & \frac{\gamma_{T}}{\kappa k^{2} R^{1 / 3}}\left\{C_{T} e^{k z}+e^{K z}\left[C_{1} e^{K z}+2\left(C_{1}+\frac{\sqrt{3}}{4} \tau\right)\right.\right. \\
& \left.\times \cos \sqrt{3} K z+\frac{\tau}{2} \sin \sqrt{3} K z\right] \cos (k x), \\
s= & \frac{\gamma_{s}}{\chi k^{2} R^{1 / 3}}\left\{\xi^{-1} C_{T} e^{k z}+e^{K z}\left[C_{1} e^{K z}+2\left(C_{1}+\frac{\sqrt{3}}{4} \tau\right)\right.\right. \\
& \left.\left.\times \cos \sqrt{3} K z+\frac{\tau}{2} \sin \sqrt{3} K z\right]\right\} \cos (k x)
\end{aligned}
$$

(в [2] в последних двух формулах вместо последнего косинуса был набран знак синуса). Здесь

$$
\begin{gathered}
K=k \mathrm{R}^{1 / 6} / 2, \quad \tau=E k / 2 \sqrt{3} K^{2} v \rho_{0}=2 E / \sqrt{3} k v \rho_{0} \mathrm{R}^{1 / 3}, \\
C_{T}=-\left(\frac{h_{T}}{h_{s}}-1\right) \Phi, \\
C_{1}=-\left[\left(1+k h_{T}\right)\left(1+\frac{1}{2 K h_{s}}\right)-\xi^{-1} \frac{h_{T}}{h_{s}}\left(1+k h_{s}\right)\right. \\
\left.\times\left(1+\frac{1}{2 K h_{T}}\right)\right] \Phi, \\
C_{1}+\frac{\sqrt{3}}{4} \tau=\frac{1}{4 K h_{s}}\left[1+k h_{T}-\xi^{-1}\left(1+k h_{s}\right)\right] \Phi, \\
\Phi=\frac{\sqrt{3}}{4} \tau\left[\left(1+k h_{T}\right)\left(1+\frac{3}{4 K h_{s}}\right)\right. \\
\left.-\xi^{-1} \frac{h_{T}}{h_{s}}\left(1+k h_{s}\right)\left(1+\frac{3}{4 K h_{T}}\right)\right]^{-1} .
\end{gathered}
$$

В [2] в последней формуле имеются две опечатки.

Решение зависит от ряда безразмерных параметров, в частности, $\mathrm{R}, \xi, \delta, b_{T} \equiv k h_{T}, b_{s} \equiv k h_{s}$ (два последних обратны соответствующим аналогам числа Био [7]). Зависимость от глубины решения для составляющих скорости (а также возмущений давления и плотности 
среды) представляет собой линейную комбинацию трех функций

$$
\exp (2 K z), \quad \exp (K z) \cos (\sqrt{3} K z), \quad \exp (K z) \sin (\sqrt{3} K z),
$$

где определенная выше величина $K \gg L^{-1}$. Экспоненты в них убывают с глубиной на масштабах $h$ порядка $L / \mathrm{R}^{1 / 6}$. В рассматриваемом приближении $\mathrm{R} \gg 1$ это много меньше характерного горизонтального масштаба возмущений $L \equiv 2 \pi / k$. Длина волны синусоид в упомянутых функциях (16) - того же порядка $h$. Этот результат аналогичен известным решениям для обычных однокомпонентных сред [1], в которых возмущения представляют собой систему циркуляционных ячеек с вертикальным и горизонтальным масштабами $h$ и $L$ соответственно, интенсивность которых экспоненциально затухает с глубиной с тем же характерным масштабом порядка $h$.

Итак, возникающие у поверхности циркуляционные ячейки, поля возмущений скорости, давления и плотности проникают в устойчиво стратифицированную среду очень неглубоко по сравнению с их горизонтальным масштабом $L$. Но в выражениях для возмущений температуры и концентрации примеси, вообще говоря, присутствует еще одно слагаемое, пропорциональное медленно убывающей экспоненте $\exp (k z)$. Это не имеет аналогов в случае однокомпонентной среды и принципиально меняет свойства решений для возникающих температурных возмущений.

Благодаря эффекту термодиффузии термические возмущения (как и возникающие возмущения концентрации примеси) могут проникать в двухкомпонентную устойчиво стратифицированную среду гораздо глубже, чем в однокомпонентную - до глубин порядка горизонтальных масштабов неоднородных напряжений $L$. При отсутствии термодиффузии $\left(s_{T}=0\right)$ коэффициент при упомянутой медленно убывающей экспоненте в полученном решении обращается в нуль, так же как и в случае одинаковых краевых условий для двух субстанций $\left(h_{T}=h_{s}\right)$. На глубинах, бо́льших $h$, возмущения температуры и концентрации примеси в большой степени компенсируют друг друга в поле плотности. Поэтому возмушения плавучести, давления и скорости проникают и в двухкомпонентные среды лишь до глубин порядка $h$ - как и в случае однокомпонентных сред.

Рассмотрим численный пример, отвечающий возможным лабораторным экспериментам. Пусть неоднородные касательные напряжения приложены к поверхности воды, устойчиво стратифицированной по температуре; $L=0.5 \mathrm{~m}, k=2 \pi / L \approx 12.5 \mathrm{~m}^{-1}, \gamma_{T}=30 \mathrm{~K} / \mathrm{m}$, $v=10^{-6} \mathrm{~m}^{2} / \mathrm{s}, \kappa=1.4 \cdot 10^{-7} \mathrm{~m}^{2} / \mathrm{s}, \alpha=3 \cdot 10^{-4} \mathrm{~K}^{-1}$, фоновая стратификация солености отсутствует. В этом случае $\mathrm{R}_{T} \approx 2.63 \cdot 10^{7}$. Имеет смысл сравнить стационарные температурные профили с учетом и без учета эффекта термодиффузии. Пусть фоновая соленость воды составляет 30\% (промилле) - порядок величины, характерный для морской воды;

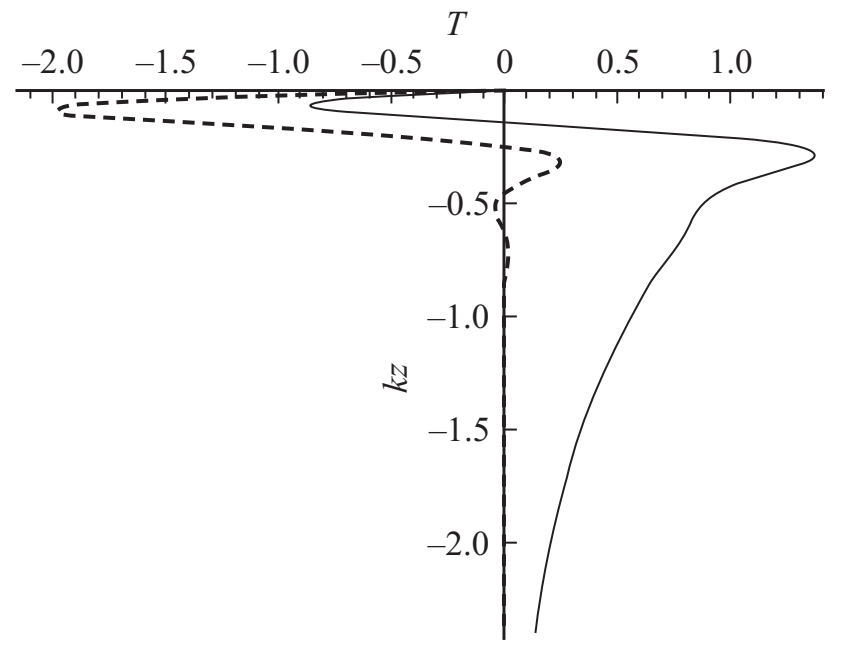

Рис. 2. Примеры безразмерных (нормированных на $\left.\sqrt{3} \cdot 10^{-3} \gamma_{T} \tau / 4 \kappa k^{2}\right)$ вертикальных профилей температурных возмущений на вертикали $x=0$ для рассмотренных в тексте численных значений параметров с учетом термодиффузии (сплошная кривая) и без ее учета $\left(\gamma_{s}=0\right.$, штриховая линия).

$\beta=0.76 \cdot 10^{-3}(\% \text { o })^{-1}, \chi=1.5 \cdot 10^{-9} \mathrm{~m}^{2} / \mathrm{s}$ [3]. Температурная зависимость коэффициента Соре $s_{T}$ приведена, например, в работе [5]. При температуре $30^{\circ} \mathrm{C}$ значение этого коэффициента порядка $10^{-3} \mathrm{~K}^{-1}$. При таких значениях параметров величины $\gamma_{s} \approx-0.94 \cdot 10^{-2} \mathrm{ppm} / \mathrm{m}$, $\mathrm{R}_{s} \approx 2.13 \cdot 10^{4}, \xi=-0.074$, так что $\mathrm{R} \approx \mathrm{R}_{T} \approx 3 \cdot 10^{7}$, $\mathrm{R}^{1 / 6} \approx 17$. На рис. 2 представлены профили температуры при $b_{T}=0, b_{s}=\infty$, рассчитанные с учетом и без учета термодиффузии. Видно, что в первом случае глубина проникновения термических возмущений в среду существенно больше (показатели основных убывающих с глубиной экспонент различаются примерно на порядок).

Этот результат можно понять из следующих соображений. В однокомпонентной среде термические возмущения не могут проникать в среду глубже масштаба $h \ll L$, поскольку на бо́льших вертикальных масштабах возникающие отклонения плавучести вызывают возникновение переносящих тепло вертикальных течений, компенсирующих температурные возмущения. Иными словами, в устойчиво стратифицированной среде существуют известные отрицательные обратные связи, благодаря которым изопикны (поверхности равной плотности) могут отклоняться от горизонтали только в тонком слое вблизи приложенной силы. При наличии же отклонений концентрации примеси последние могут компенсировать температурные отклонения в поле плотности - выравнивание изопикн не исключает возможности возникновения значительных отклонений в поле каждой из этих субстанций, что и происходит в рассмотренном примере. Таким образом, „совместные“ отклонения температуры и концентрации могут глубоко проникать в среду (до глубин порядка $L$ ), не вызывая отклонений плавучести и упомянутых компенсирующих течений. 


\section{Заключение}

В геофизической гидродинамике эффекты термодиффузии обычно не учитываются. Считается, что при реальной (относительно небольшой) солености морской воды они незначительны ввиду малости значений коэффициента Соре $s_{T}$ и фоновой концентрации примеси $\bar{\mu}$. Но представленные расчеты показывают, что в некоторых ситуациях эти эффекты могут быть заметными. Роль возникающих отклонений концентрации примеси становится заметной благодаря двум обстоятельствам: 1) медленность диффузии примеси, что способствует „жизнеспособности“ отклонений ее концентрации; 2) различие краевых условий для температуры и концентрации на верхней границе - в рассмотренном численном примере температура на поверхности жестко фиксирована, в то время как отклонения концентрации примеси не теряются на границе.

Еще более значительными эффекты термодиффузии могут быть в средах с бо́льшими концентрациями примеси или с бо́льшими значениями коэффициента Соре. Один из возможных примеров - „солнечные пруды“ (solar ponds [5]) - искусственные водоемы, используемые для аккумулирования солнечной энергии. Конвективный теплообмен в них подавляется путем существенного искусственного повышения солености в придонных слоях - поддержания устойчивой стратификации плотности.

В рассмотренном примере значение коэффициента Соре было положительным. Но оно может быть и отрицательным. Например, в соленой воде его значение отрицательно при более низких температурах - меняет знак при температуре около $10^{\circ} \mathrm{C}$ [5]. В этом случае значения параметров $\gamma_{s}$ и $\xi$ положительны, и выражение в квадратных скобках в (15), вообще говоря, может приближаться к нулю, что соответствует неограниченному возрастанию амплитуды возмущений - порогу неустойчивости. Такой тип неустойчивости, обусловленный термодиффузией, насколько нам известно, ранее не рассматривался. Условие неустойчивости с точностью до обозначений совпадает с [2], где неустойчивость обусловлена не термодиффузией, а наличием фоновой стратификации концентрации примеси. Это условие подробно проанализировано в упомянутой работе.

\section{Список литературы}

[1] Линейкин П.С., Мадерич В.С. Теория океанического термоклина. Л.: Гидрометеоиздат, 1982. $271 \mathrm{c.}$

[2] Ингель Л.Х. // ЖЭТФ. 2006. Т. 129. № 1. С. 213-220.

[3] Ингель Л.Х., Калашник М.В. // УФН. 2012. Т. 182. № 4. C. 379-406.

[4] Рыжкков И.И. Термодиффузия в смесях: уравнения, симметрии, решения и их устойчивость. Новосибирск: СО РАН, 2013. $200 \mathrm{c}$.

[5] Angeli C., Leonardi E. // Int. J. Heat Mass Transfer. 2005. Vol. 48. P. 4633-4639.
[6] Гершуни Г.3., Жуховицкий Е.М. Конвективная устойчивость несжимаемой жидкости. М.: Наука, 1972. 392 с.

[7] Карташов Э.М. Аналитические методы в теории теплопроводности твердых тел. М.: Высшая школа, 2001. 550 с. 